\title{
A Proximity and Pressure Touch Screen Using Mutual Capacitance Measurement
}

\author{
Satoshi Tsujia,", Teruhiko Kohama ${ }^{\mathrm{a}}$ \\ aFukuoka University, 8-19-1 Nanakuma, Jonan-ku, Fukuoka 814-0180, Japan, \\ *Corresponding Author: tsuji@fukuoka-u.ac.jp
}

\begin{abstract}
Touch screens have become one of the most common human interfaces in recent year. Most touch screens detect contact position only. We proposed the proximity and pressure touch screen method. This touch screen method consists of plane separation-type electrodes and parallel-type electrodes with mutual capacitance measurement. In this paper, we describe a proposed sensor using indium tin oxide (ITO) electrodes which are transparent on the LCD monitor. In addition, we consider the precise $\mathrm{X}-\mathrm{Y}-\mathrm{Z}$ position measurement by the proposed touch screen using the correction processing. The prototype sensor using this technique detects an object and its position on the LCD monitor before and after contact. We think that the proposed touch screen will be a functional and useful interface.
\end{abstract}

Keywords: Touch screen, Proximity and pressure sensor, Mutual capacitance measurement.

\section{Introduction}

In recent year, touch screens have become one of the most common human interfaces for electronics devices. For small touch screen such as smartphones and tablet device, mutual capacitance measurement has been used mainly because it can detect multi-touch events ${ }^{(1)}$. Most of previous touch screen only detect the contact position between the touch screen and the object.

On the other hand, proximity touch screens to detect a finger before contact have been proposed. The method using a camera to detect the finger at proximity range has also been proposed ${ }^{(2)}$. However, this device become large because an external camera is required. Thus, it is difficult to use for small mobile devices. For small mobile devices, a method combining self-capacitance measurement and mutual capacitance measurement to detect fingers within the proximity range and its contact position has been presented (3). However, it is difficult to detect multi-point touch using self-capacitance measurement because 'ghosts' occur ${ }^{(1)}$, and it is not considered to detect pressure.

On the other hand, the pressure touch screens to detect the contact pressure have been proposed. The touchpad for force sensing is proposed using resistance measurement ${ }^{(4)}$. The methods using some pressure sensor attaching the smartphone to detect the pressure condition is proposed ${ }^{(5)}$. In addition, the smartphone ${ }^{(6-7)}$ which can detect the contact position and pressure using pressure sensor and touch screen were on sale. However, it is not considered to detect the object at proximity range.

The objective of our study is to develop a proximity and pressure touch screen using mutual capacitance measurement to detect an object within the proximity range, contact position, and pressure to operate the display of the device. Fig. 1 shows the objective image of the propose proximity and pressure touch screen on a LCD monitor. When a finger approaches the touch screen, the touch screen detects the finger position before the contact, and the displayed image is enlarged according to the position of the

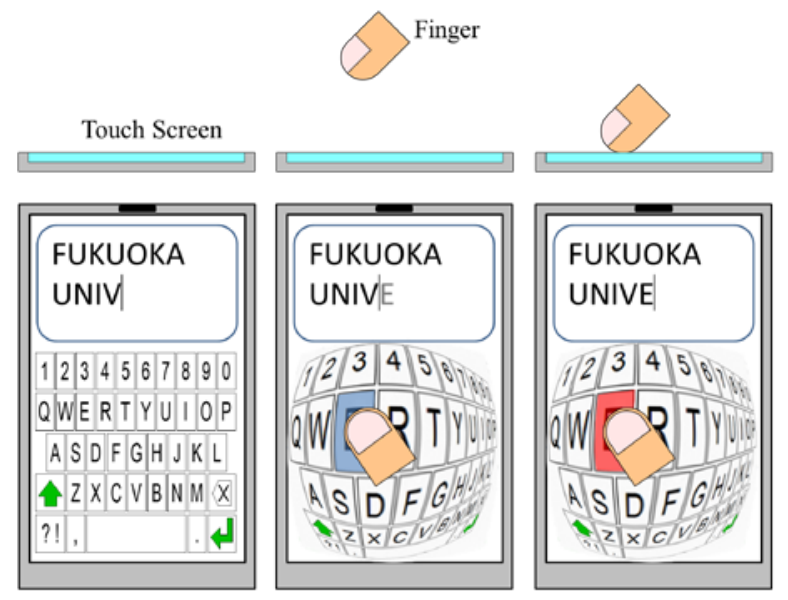

Fig. 1. The objective image of proximity and pressure touch screen in this study. 
finger. Thus, it is easy to push small interface buttons. When the finger touches the sensor, the touch screen and pressure sensor detect the pressure and its position of the object, and the image is operated by applying a relatively pressure. Therefore, this sensor would assure the functionality and usability of devices.

Previously, we proposed a layered 3D touch screen to detect an object both before and after contact ${ }^{(8)}$. In the sensor, two types of capacitance sensors which are the plane-type and parallel-type capacitance sensor are layered on the LCD monitor. The size of plane-type capacitance electrodes of the top sensor is large to increase sensitivity before contact, and the size of parallel-type capacitance electrodes of the bottom sensor are small to realize a high spatial resolution during contact. However, the measurement points of the top sensor are few because the electrodes of the top sensor are large. Thus, the resolution is reduced within the proximity range.

In addition, we proposed a proximity and pressure touch screen method using separation-type electrodes with mutual capacitance measurement ${ }^{(9)}$. The sensor consists of the separate-type sensor and the capacitance pressure sensor which is parallel-type electrodes. The proposed sensor can combine and separate electrodes on separate-type sensor at proximity range. The coupled electrodes are large to increase the sensitivity along the Z-axis, and the separated electrodes increase the resolution of the $\mathrm{X}-\mathrm{Y}$ axes when the object is in close proximity range. In addition, the capacitance pressure sensor detects the pressure and center position on contact. However, the electrodes of the proposed sensor are copper plate, which is not transparent.

In this paper, we describe a proposed sensor using indium tin oxide (ITO) electrodes which are transparent on the LCD monitor. In addition, we consider the precise X-Y$\mathrm{Z}$ position measurement by the proposed touch screen using the correction processing. The prototype sensor using this technique detects an object and its position on the LCD monitor before and after contact.

\section{Principles}

Fig. 2 (a) shows the schematic diagram of the proposed method. The proposed sensor consists of plane-type electrodes $\left(E_{1}\right.$ and $\left.E_{2}\right)$ and four parallel-type electrodes which is the pressure sensor. The plane-type electrodes detect the finger position and multi-point of the object within the proximity range. The parallel-type electrodes detect the pressure and precise center point of the touch ${ }^{(9)}$. In the proposed method, the coupled electrodes which are large can
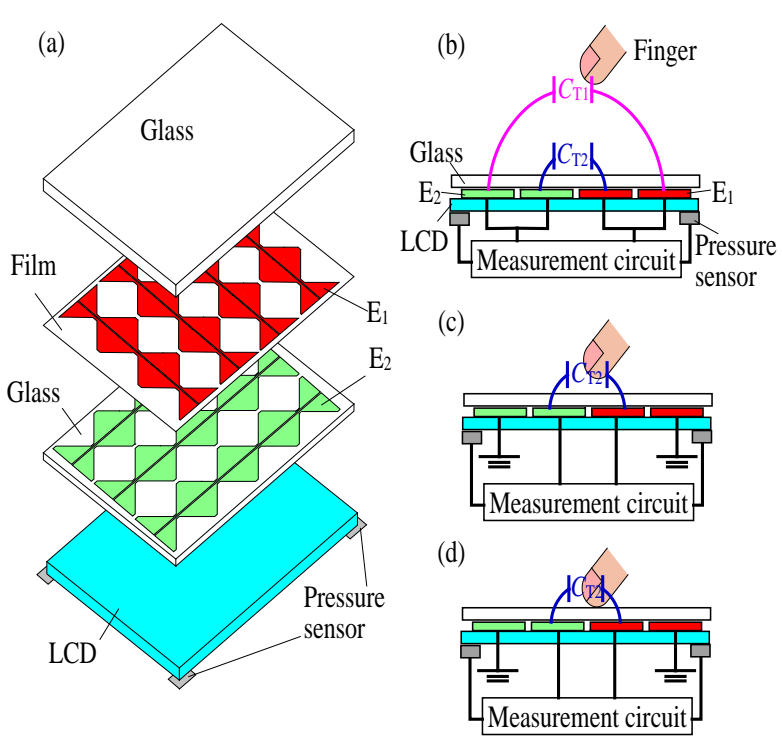

Fig. 2. Principle of the proposed proximity and pressure touch screen. (a) Schematic diagram of proximity and pressure touch screen. (b) Measurement of the coupled electrode. (c) Measurement of the separated electrode. (d) Measurement of the pressure sensor.

be separated at the center point ${ }^{(10)}$. Fig. 2(b) shows the measurement of the coupled electrodes, and Fig. 2(c) shows the measurement of the separated electrodes. In Fig. 2(b), the coupled electrodes measure the capacitance $(C)$. $C_{T}$ is given by

$$
C_{T}=C_{T 1}+C_{T 2}
$$

Here, $C_{T 1}$ is the capacitance between far-spaced electrodes, and $C_{T 2}$ is the capacitance between closely spaced electrodes. When the object is far away, mainly $C_{T 1}$ is changed. Thus, the proposed method can detect an object far away from the sensor because coupled electrodes can detect the change in $C_{T 1}$. In addition, $C_{T 2}$ is changed when the object is near. Thus, it is possible to increase the $\mathrm{X}-\mathrm{Y}$ resolution with separated electrodes because the separation between the electrodes is small. $C_{T}$ of the nearest electrodes from an object is changed most when an object approaches the sensor, and the variation of $C_{T}\left(\Delta C_{T}\right)$ of electrodes further away from an object is small. Thus, we calculated the $\mathrm{X}-\mathrm{Y}$ position of an object in the proximity range from the distribution of $\Delta C_{T}$ for each electrode. $\mathrm{X}$ position $\left(X_{T P}\right)$ was calculated by the following equation using $\Delta C_{T}$ :

$$
X_{T P}=\frac{\sum_{k=A-1}^{A+1} X_{k} \cdot \Delta C_{T M k}}{\sum_{k=A-1}^{A+1} \Delta C_{T M k}}
$$

Here, $\Delta C_{T M A}$ is $\Delta C_{T}$ of the most changing value and $X_{A}$ is the electrode position of $\Delta C_{T M A}$ along the X-axis. Thus, it is 
possible to detect the center position of a finger on $X_{A}$ using $\Delta C_{T M A}$ and $\Delta C_{T}$ on both sides of $X_{A}{ }^{(10)}$.

In addition, four parallel-type electrodes detect the pressure and a precise center point of the touch. We calculated the $\mathrm{X}-\mathrm{Y}$ position of the object on the contact using $\Delta C_{B}$ of each parallel-type electrode. The $\mathrm{X}\left(X_{B P}\right)$ and $\mathrm{Y}\left(Y_{B P}\right)$ positions were calculated by the following equations.

$$
\begin{gathered}
X_{B P}=\frac{\Delta C_{B 1}-\Delta C_{B 2}-\Delta C_{B 3}+\Delta C_{B 4}}{\Delta C_{B 1}+\Delta C_{B 2}+\Delta C_{B 3}+\Delta C_{B 4}} \\
Y_{B P}=\frac{\Delta C_{B 1}+\Delta C_{B 2}-\Delta C_{B 3}-\Delta C_{B 4}}{\Delta C_{B 1}+\Delta C_{B 2}+\Delta C_{B 3}+\Delta C_{B 4}}
\end{gathered}
$$

Here, $\Delta C_{B 1}-\Delta C_{B 4}$ are the capacitance variations of each parallel-type sensor $\left(\mathrm{S}_{1}-\mathrm{S}_{4}\right)$. In addition, we corrected $X_{B P}$ by the least square method using (5).

$$
X_{B P}{ }^{\prime}=k_{0}+k_{1} X_{B P}+k_{2} X_{B P}{ }^{2}+k_{3} X_{B P}{ }^{3}
$$

Here, $k_{i}(i=0-3)$ denote the coefficients. The pressure $(P)$ was calculated by the following least square method using the values of each parallel-type sensor $\left(\mathrm{S}_{1}-\mathrm{S}_{4}\right)$.

$$
P=f\left(\Delta C_{A}\right)+f(D)
$$

Here, $\Delta C_{A}$ is the sum of $\Delta C_{B 1}-\Delta C_{B 4}$, and $D$ is the distance from the center of the electrodes where the capacitance displayed the largest change. Thus, the proposed sensor can detect the object using coupled electrodes when an object is far away, and can detect the object using separated electrodes when the object is near. The proposed sensor can detect the multi point using separated electrodes, and can detect the pressure and a precise center point using parallel-type electrodes on contact ${ }^{(9)}$.

\section{Prototype Touch Screen}

Fig. 3 show the structure of the prototype touch screen using ITO electrodes on the LCD monitor. We proposed a structure wherein the coupled electrodes $\left(E_{1}\right.$ and $\left.E_{2}\right)$ of the plane-type capacitance sensor are large to increase the sensitivity before contact. In addition, the electrodes can be separated to realize a high spatial resolution at close proximity range. Thus, the prototype plane sensor consists of $4 \times 3$ coupled electrodes and $8 \times 6$ separated electrodes ${ }^{(10)}$. The electrode patterns $\left(E_{1}\right.$ and $E_{2}$ ) are located on the first ITO sheet, which includes a glass sheet. The electrodes are connected by a jumper pattern (J) on the second sheet. A glass sheet (Thickness is $0.7 \mathrm{~mm}$ ) denoted by Gl in Fig. 3, is placed on top of the sensor to isolate it from objects. An overcoat layer (Thickness is $1.5 \mu \mathrm{m}$ ) denoted by OC in Fig. 3 , is placed between $E_{1}$ and $E_{2}$ to isolate the electrodes. The shield electrode (Sh) is set between the plan-type electrodes

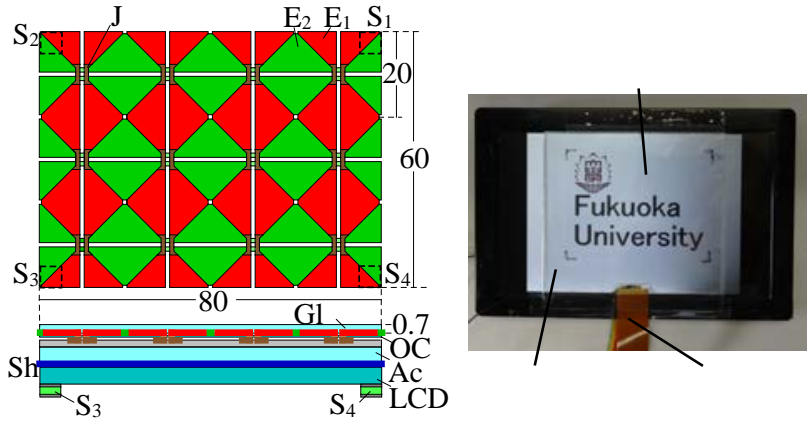

Fig. 3. Sensor structure. $E_{1}$ and $E_{2}$ : Electrodes, $S_{1}-S_{4}$ : Pressure sensor, J: Jumper, Ac: Acrylic, Sh: Shield electrodes, Gl: Glass, LCD: LCD monitor, and OC: Overcoat layer. Dimensions are in $\mathrm{mm}$.

and the LCD monitor. The parallel-type electrodes are set at the four corners on the bottom of LCD monitor. The size of electrodes of parallel-type electrodes are $5 \mathrm{~mm} \times 5 \mathrm{~mm}$. The urethane gel (Thickness is $1 \mathrm{~mm}$ ) is set between parallel-type electrodes. The capacitances are measured by a control circuit which consists of an analog switch, microcomputer, and capacitance measurement IC (Analog Devices, AD7142). The AD7142 is a capacitance-to-digital converter (16-bit), and the unit of the measurement values indicates $A / D$ converted digital values (digit). The measured data (16-bit) are captured by the PC. The electrodes not used for measurement are connected to a shield, which is either the ground or has an equal potential to that of the measurement signal. The object is a grounded conductor which is the finger model in this study. The object was attached to a robot arm.

\section{Results and Discussion}

\subsection{Z Position Measurement}

We consider the individual difference of the object at proximity measurement because the capacitance changes according to the object characteristics. In this experiment, the object size $(\varphi)$ are $10 \mathrm{~mm}, 15 \mathrm{~mm}$ and $20 \mathrm{~mm}$ in Fig. 4. Fig. 4 show the measurement condition. Here, the color electrodes are measurement electrodes. Fig. 5 shows the results of each $\Delta C_{T}$. In Fig. 5, $\Delta C_{T}$ changes according to distance ( $d$ ) between sensor and object. $\Delta C_{T}$ in the coupled sensor is changed from far away, and $\Delta C_{T}$ in separated electrodes is changed from nearby. This is the reason for the increased detection sensitivity along the Z-axis when increasing the size of the electrodes. In addition, $\Delta C_{T}$ changes according to the object size. Thus, it is difficult to detect the $\mathrm{Z}$ position from $\Delta C_{T}$ when the electrical characteristics of a finger is unknown. However, we think 


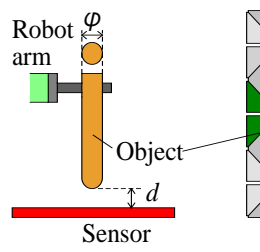

(a)

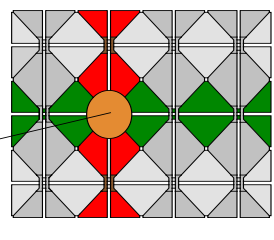

(b)

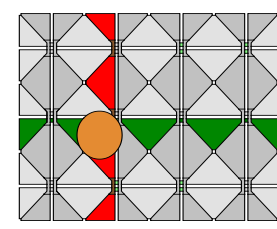

(c)
Fig. 4. Measurement condition. (a) The distance condition. (b) The condition of coupled electrodes. (c) The condition of separated electrodes.

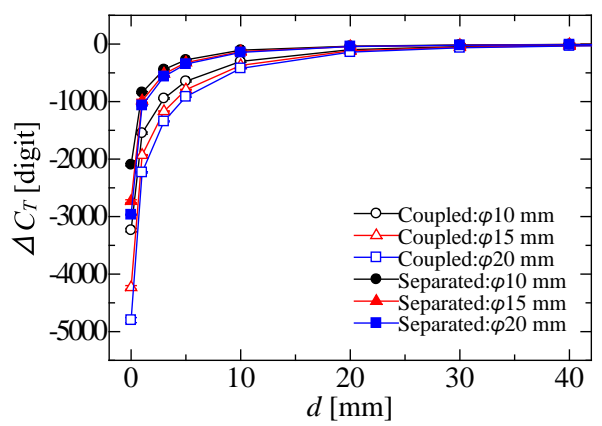

Fig. 5. Relationship between the distance (d) and $\Delta C$.

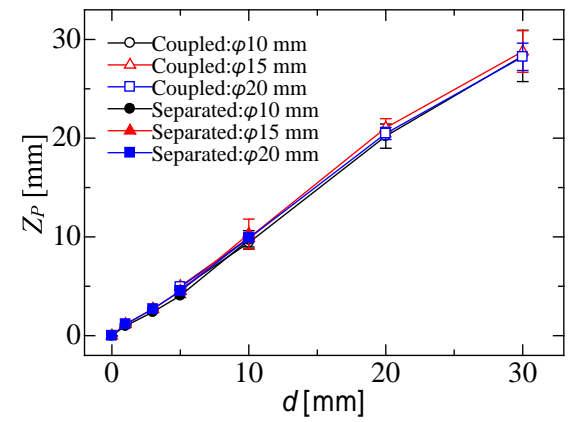

Fig. 6. Relationship between the $d$ and $Z_{P}$.

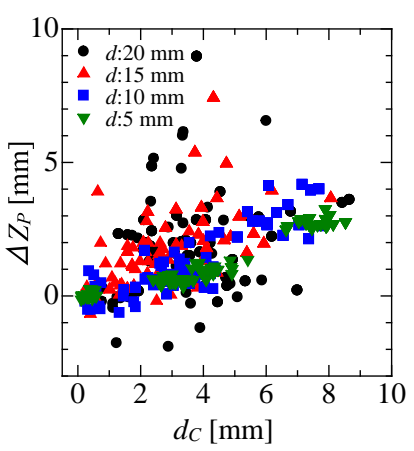

(a)

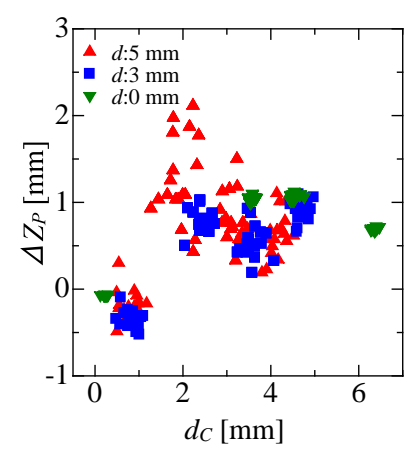

(b)
Fig. 7. Relationship between the $d_{C}$ and $\Delta Z_{P}$. (a) The results of coupled electrodes. (b) The results of separated electrodes.

that the sensor can detect the $\mathrm{Z}$ position using the electrical characteristic. The electrical characteristic of the finger is measured by this sensor when the object touch, and the touch condition can be detected by pressure sensor. In this experiment, $\Delta C_{T}$ was corrected by the ratio with each capacitance on the contact ( $0 \mathrm{~mm}$ ) based on the experimental results of $\varphi 15 \mathrm{~mm}$. In addition, we calculated the Z-axis position $\left(Z_{P}\right)$ using the least squares method. $Z_{P}$ is given by

$$
Z_{P}=\frac{1}{k_{4}+k_{5} \Delta C_{T M A}+k_{6} \Delta C_{T M A}{ }^{2}}
$$

Here, $k_{i}(i=4-6)$ indicates the coefficients. Fig. 6 shows calculated $Z_{P}$ using Eq. (7) at proximity range. $Z_{P}$ changes according to $d$. Thus, we think that the sensor can detect the Z-axis position using Eq. (7). However, $\Delta C_{T}$ changes according to $\mathrm{Z}$ position and $\mathrm{X}-\mathrm{Y}$ position. Thus, we consider the influence of $X-Y$ position on $Z$ position measurement. Fig. 7 shows the relationship between $\Delta Z_{P}$ and $d_{C}$ which is $\mathrm{X}-\mathrm{Y}$ distance between the center point of the electrodes of $C_{T M A}$ and the object. The object size $(\varphi)$ is $15 \mathrm{~mm}$. Here, $\Delta Z_{P}$ is the difference between the $Z_{P}$ and the theoretical value of $Z$ position. $d_{C}$ was obtained Eq. (2). We corrected $Z_{P}$ using $\mathrm{X}-\mathrm{Y}$ position by the least squares method. The corrected $\mathrm{Z}$ position $\left(Z_{P}{ }^{\prime}\right)$ is given by

$$
Z_{P}{ }^{\prime}=Z_{P}+f\left(d_{C}\right)
$$

Here, $f\left(d_{C}\right)$ was obtained by the quadratic least squares method. Thus, we think that the sensor can detect the $\mathrm{Z}$ position using Eq. (8).

\subsection{Measurement of X-Y Position}

We show the results of $\mathrm{X}-\mathrm{Y}$ position measurement with the proposed coupled electrodes and separated electrodes at proximity range. The object size $(\varphi)$ is $15 \mathrm{~mm}$. We calculated the $\mathrm{X}-\mathrm{Y}$ axis position using Eq. (2). The object was moved onto an arrow $\pm 10 \mathrm{~mm}$ from the center of the electrode in 2 $\mathrm{mm}$ increments in the $\mathrm{X}$ direction (Fig. 8). Fig. 9 shows $X_{T P}$ using Eq. (2). The theoretical position value was the object position using the robot arm. In Fig. 9, $X_{T P}$ is changed according to the $X$ position of the object. The results are not high accuracy of $X-Y$ position. Thus, we correct the $X-Y$ position using the least squares method. The corrected $X$ position $\left(X_{T P}{ }^{\prime}\right)$ is given by

$$
X_{T P}{ }^{\prime}=k_{7}+k_{8} f\left(Z_{P}^{\prime}\right) X_{T P}
$$

Here, $k_{i}(i=7-8)$ indicates the coefficients. $f\left(Z_{P}{ }^{\prime}\right)$ was obtained by the least squares method. Fig. 10 shows calculated $X_{T P}$ ' using Eq. (9) at proximity range. In Fig. 10, $X_{T P}$ ' is changed according to the $\mathrm{X}$ position of the object. Thus, it is possible to improve the accuracy by correlation using the least squares method (Eq. (9)) for each electrode interval. 


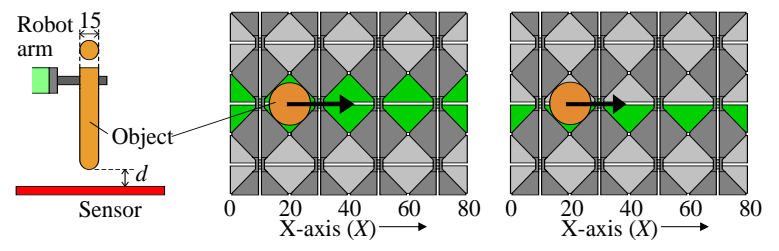

Fig. 8. Measurement condition.

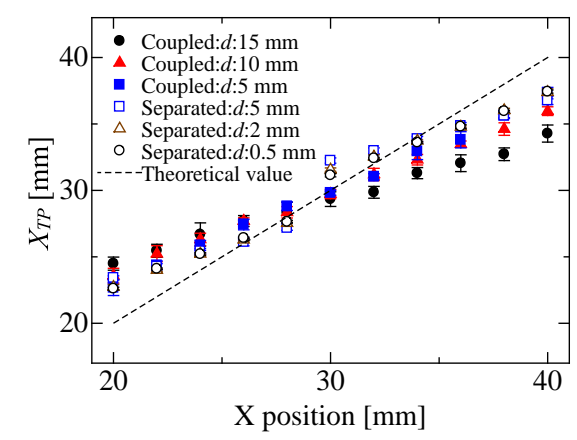

Fig. 9. Relationship between the $\mathrm{X}$ position and $X_{B P}$.

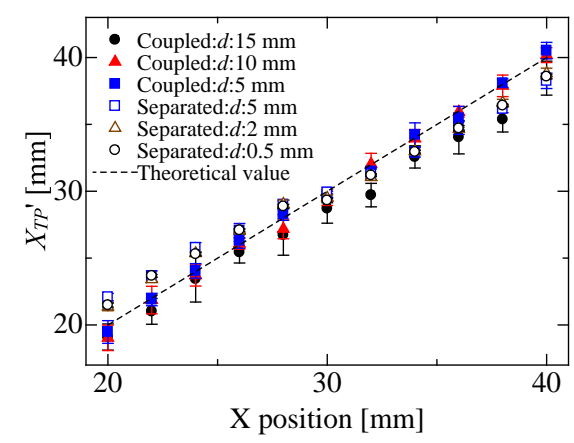

Fig. 10. Relationship between the $\mathrm{X}$ position and $X_{B P}$ '.

\subsection{Measurement of X-Y-Z Position}

We evaluated the X-Y-Z position measurement. Fig. 11 shows the measurement condition. The object size $(\varphi)$ is 15 $\mathrm{mm}$. $d$ are $17 \mathrm{~mm}, 12 \mathrm{~mm}, 7 \mathrm{~mm}$ and $2 \mathrm{~mm}$, and the pressures are $3 \mathrm{~N}$ and $7 \mathrm{~N}$. Fig. 12(a) shows the calculated $\mathrm{X}-\mathrm{Y}$ coordinate position. Fig. 12(b) shows the calculated $\mathrm{Z}$ position and pressure. The $\mathrm{X}-\mathrm{Y}-\mathrm{Z}$ position and pressure can be detected using this proposed sensor. In this experiment, the average $\mathrm{X}-\mathrm{Y}$ position error at $17 \mathrm{~mm}$ was $2.7 \mathrm{~mm}$, that at $12 \mathrm{~mm}$ was $1.0 \mathrm{~mm}$, that at $7 \mathrm{~mm}$ was $1.6 \mathrm{~mm}$, that at $2 \mathrm{~mm}$ was $0.76 \mathrm{~mm}$, that at $3 \mathrm{~N}$ was $3.4 \mathrm{~mm}$, and that at $7 \mathrm{~N}$ was $3.7 \mathrm{~mm}$. The average $\mathrm{Z}$ position error at $17 \mathrm{~mm}$ was $1.6 \mathrm{~mm}$, that at $12 \mathrm{~mm}$ was $1.3 \mathrm{~mm}$, that at $7 \mathrm{~mm}$ was $0.31 \mathrm{~mm}$, that at $2 \mathrm{~mm}$ was $0.34 \mathrm{~mm}$. The average pressure error at $3 \mathrm{~N}$ was $0.087 \mathrm{~N}$, that at $7 \mathrm{~N}$ was $0.25 \mathrm{~N}$. The average $\mathrm{X}-\mathrm{Y}$ position error on pressure sensor was large. This main cause is that the weight distribution of LCD monitor is not uniform, and pressure sensor is behind LCD monitor. We will try to improve the accuracy in the future work.

We evaluated the proximity and contact measurement using the proposed sensor. Fig. 13 shows the results. Fig.

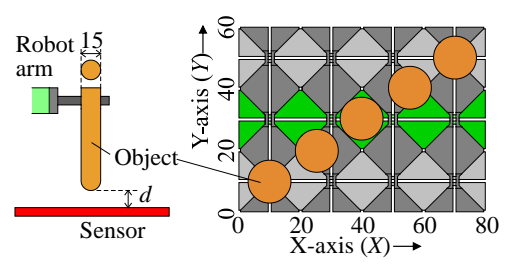

Fig. 11. Measurement condition.

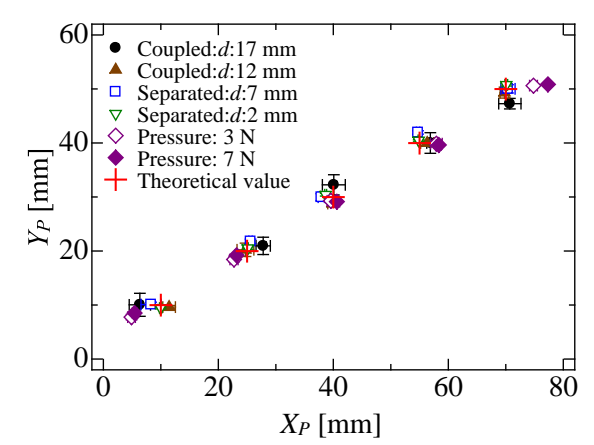

(a)

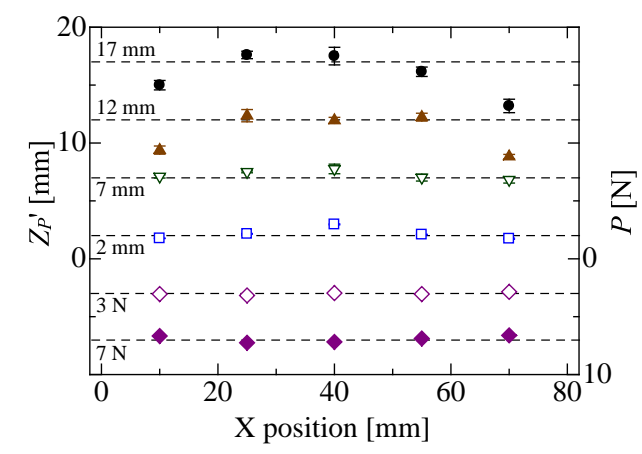

(b)

Fig. 12. Measurement results. (a) Relationship between $X_{B P}$ ' and $Y_{B P}$ '. (b) Relationship between $X$ position and, $Z_{P}$ ' and $P$.

13(a) shows the measurement position. A finger approached the sensor ( $\mathrm{B}$ and $\mathrm{C}$ ). The finger pushed the sensor with a weak force (D), a middle force (E) and a strong force (F). Two fingers approached the sensor $(G)$ and pushed the sensor ( $\mathrm{H}$ and I). Fig. 13(b) shows $\Delta C_{T}$ of the coupled electrodes and separated electrodes. Fig. 13(c) shows the calculated pressure and center position using the pressure sensor. Fig. 13(b) show that $\Delta C_{T}$ varied according to the finger position at the non-contact points (B to $\mathrm{C}$ ). Thus, the sensor could detect the object before contact (proximity range). Fig. 13(c) shows pressure $(P)$ and object position when the finger pushed the sensor (D - F). Thus, the sensor could detect the pressure and position. In addition, the sensor could detect multiple positions ( $\mathrm{G}-\mathrm{I}$ ) using the plane-type sensor.

Therefore, the coupled electrodes detected the object position when the object was far away. The separated electrodes detected the object position when it was near or in contact with the screen. In addition, the pressure sensor detected the pressure and its center position at the contact. 

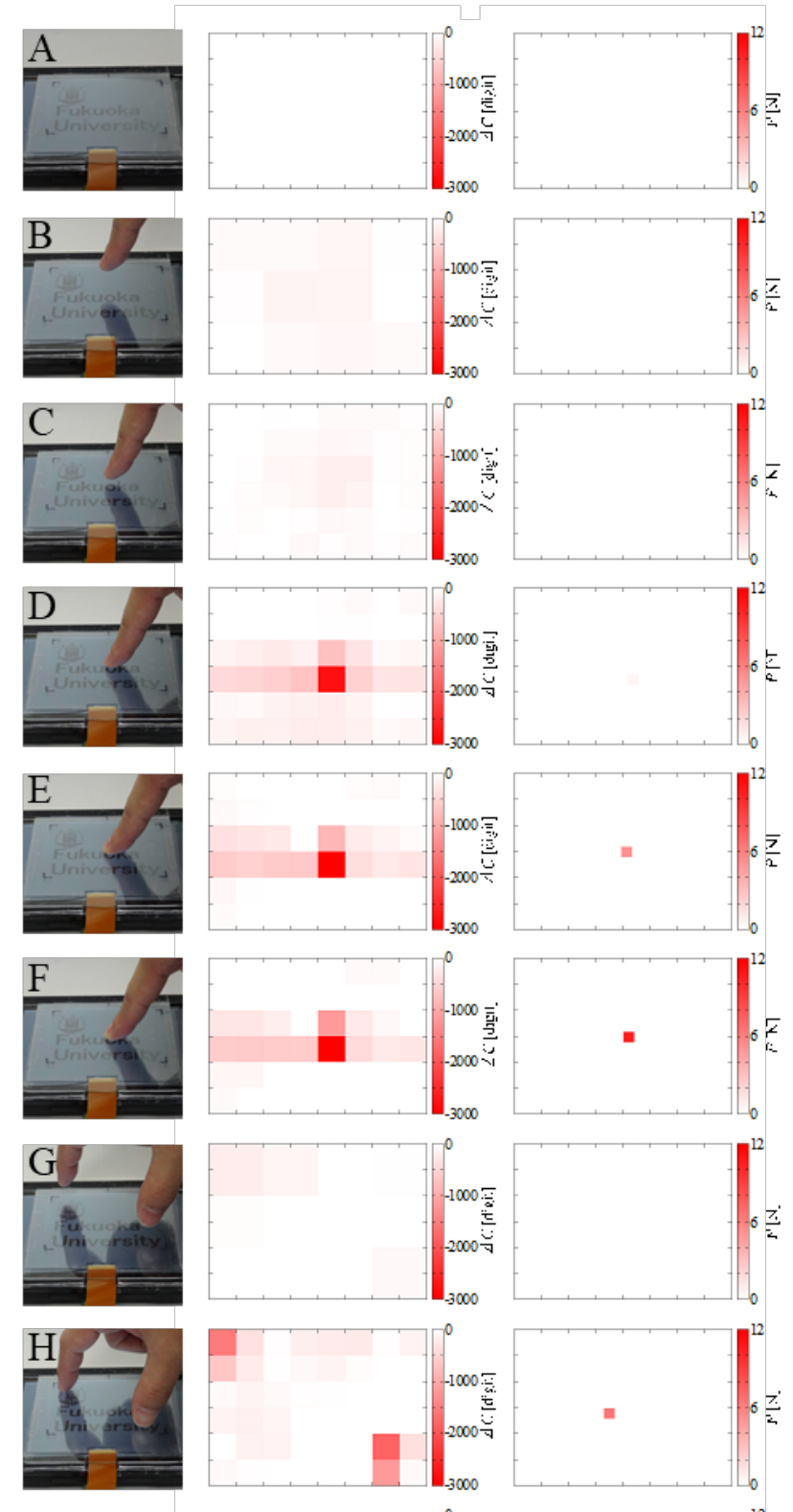

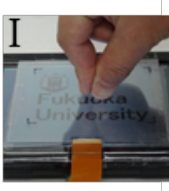

(a)

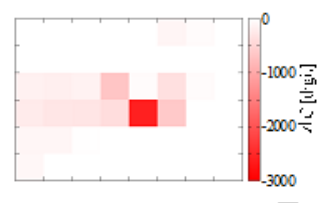

(b)
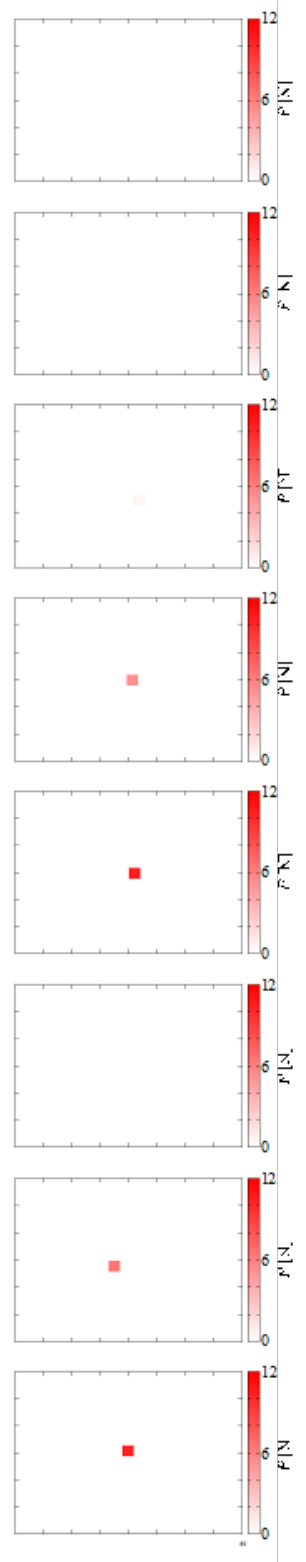

(c)

Fig. 13. Measurement results of the proposed sensors.

Here, the plane-type electrodes detected the multiple points during contact as the pressure sensor cannot detect multiple points. We think that measurement is possible from the proximity range to the contact point by complementing each other using an upper capacitive sensor and a lower pressure sensor.

\section{Conclusions}

We proposed a proximity and pressure touch screen using mutual capacitance measurement. In this paper, we describe a proposed sensor using indium tin oxide (ITO) electrodes on LCD monitor. In addition, we consider the precise $\mathrm{X}-\mathrm{Y}-\mathrm{Z}$ position measurement by the proposed proximity touch screen using the correction processing. The prototype sensor using this technique detects an object and its position on LCD monitor before and after contact. Thus, we think that the proposed sensor will be one of useful methods as a touch screen.

\section{Acknowledgment}

This work was supported in part by JSPS KAKENHI Grant Number JP 15K16043.

\section{References}

(1) G. Barrett and R. Omote : "Projected-capacitive touch technology”, Inf. Disp., Vol. 26, No. 3, pp. 16-21, 2010.

(2) O. Hilliges, S. Izadi, A. D. Wilson, S. Hodges, A. G. Mendoza, and A. Butz : "Interactions in the air: adding further depth to interactive tabletops”, Proceedings of UIST '09, pp. 139-148, 2009

(3) http://www.cypress.com/products/truetouch-touchscree n-controllers

(4) D. K. Kim, J. H. Kim, M. J. Kwon, and Y. H. Kwon : "A touchpad for force and location sensing”, ETRI J. Vol. 32, No. 5, pp. 722-728. 2010

(5) S. Heo and G. Lee : "Force gestures: augmented touch screen gestures using normal and tangential force", Proceedings of CHI EA '11, pp.1909-1914, 2011

(6) E. Furuki and Y. Kikuchi : "Approach to Commercialization of RakuRaku Smartphone", FUJITSU, Vol. 63, No. 5, pp. 548-554, 2012

(7) https://www.apple.com/jp/shop/buy-iphone/iphone6s

(8) S. Tsuji, T. Kohama : "A Layered 3D Touch Screen Using Capacitance Measurement”, IEEE Sensors Journal, Vol. 14, No. 9, pp. 3040-3045, 2014

(9) S. Tsuji, T. Kohama : "A Proximity and Tactile Touch Screen Using Separation-Type Electrodes”, Proceedings of the 5th IIAE International Conference on Intelligent Systems and Image Processing 2017, pp. 62-66, 2017

(10) S. Tsuji, T. Kohama : "A Proximity Touch Screen Using Mutual Capacitance Measurement”, Proceedings of the 2016 IEEE International Conference on Robotics and Biomimetics, pp. 1262-1266, 2016 